\title{
Smart SQUIDs based on Relaxation Oscillation SQUIDs
}

\author{
M. J. van Duuren, G. C. S. Brons, J. Flokstra and H. Rogalla \\ Low Temperature Division, Department of Applied Physics, University of Twente, P.O.Box 217, 7500 AE Enschede, The Netherlands
}

\begin{abstract}
Smart SQUIDs based on double Relaxation Oscillation SQUIDs (DROS) and a superconducting up-down counter have been developed. DROS and counter form a flux locked loop on one single chip. The DROS output consists of a series of pulses that controls the two up and down write gates of the counter. The pulsed output structure of the DROS constitutes the internal clock for this single-chip device. Several prototypes were built with a clock frequency of $100 \mathrm{MHz}$, a linear operation flux range of about $2.5 \Phi_{0}$ and a white noise level of $6.5 \mu \Phi_{0} / N H z$. The smart SQUID is in principle a promising device for application in multichannel SQUID systems.
\end{abstract}

\section{INTRODUCTION}

Multichannel SQUID systems based on non-hysteretic dc SQUIDs require a large number of wires between the roomtemperature electronics and the SQUIDs. The number of channels in biomagnetic magnetometers and SQUID-array particle detectors may be several of hundreds resulting in more than a thousand connecting lines. As a consequence, such systems have a large helium boil-off rate, are complex and have a decreased reliability. To reduce the number of wires in multichannel systems, several research groups have developed digital single-chip SQUIDs where the entire flux locked loop (FLL) circuit is integrated with the SQUID chip [1-4]. Multiplexing of the channel output signals makes a further reduction of the number of wires possible. Additional advantages of the single-chip approach are the reduced cross talk between the different channels, simpler room temperature electronics and the potential for a very large bandwidth.

Most single-chip digital SQUIDs are based on the concept developed at Fujitsu [5]. The main elements of the device are a comparator, being a hysteretic dc SQUID with asymmetric current injection driven by an external clock and a superconducting up-down counter.

We developed a single-chip SQUID with integrated flux locked loop circuit based on a Double Relaxation Oscillation SQUID (DROS) [6] and a superconducting up-down counter. We call this a Smart SQUID. The DROS itself acts as an onchip clock, which enables smooth operation at high frequencies resulting in a better sensitivity and a larger bandwidth. We describe the operation principles of the Smart SQUID and present the results of simulations and measurements on the integrated device.

Manuscript received September 15, 1998

This work was supported in part by the foundation "Stichting Technische Wetenschappen.

\section{OPERATION PRINCIPLE OF The SMART SQuid}

\section{A. DROS}

A DROS consists of two hysteretic de SQUIDs in series which are shunted by an external L-R shunt, as shown schematically in Fig. 1. The signal flux $\Phi_{\text {sig }}$ is applied to the upper SQUID (the signal SQUID) and a constant flux $\Phi_{\text {ref }}$ is applied to the other SQUID (the reference $S Q U I D$ ). If the DROS is biased with an appropriate dc bias current $I_{\mathrm{b}}$, relaxation oscillations are generated. The frequency of these oscillations, $f_{\mathrm{RO}}$, depends mainly on the time constant $L_{\mathrm{sh}} / R_{\mathrm{sh}}$ of the relaxation circuit, and amounts to $1 \mathrm{GHz}$ for most practical devices [6,7].

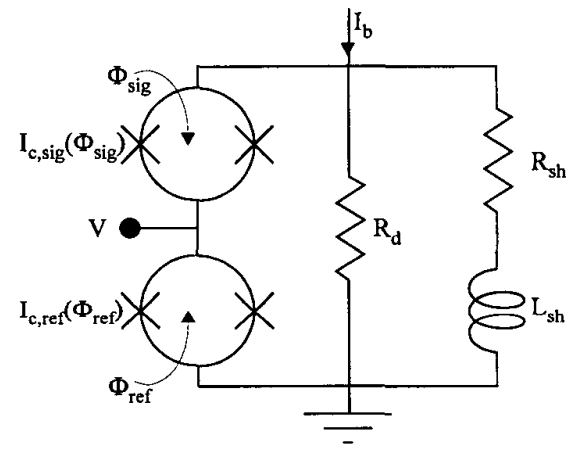

Fig. 1. Scheme of a DROS.

Only the SQUID with the lowest critical current participates in the relaxation oscillations, whereas the other SQUID remains superconducting at the time. As the critical current of the signal SQUID depends on the signal flux $\Phi_{\text {sig, }}$ it is the signal flux that determines which one of the two SQUIDs oscillates. If $I_{\mathrm{c} \text {,sig }}\left(\Phi_{\text {sig }}\right)<I_{\text {cref }}$, the reference SQUID stays superconducting and the signal SQUID oscillates, whereas, if $\Phi_{\text {sig }}$ is changed so as to make $I_{\mathrm{c}, \mathrm{sig}}\left(\Phi_{\mathrm{sig}}\right)>I_{\mathrm{c}, \mathrm{ref}}$, the reference SQUID oscillates while the signal SQUID is superconducting. This pulsed output of a DROS is particularly suited for digital readout.

\section{B. Smart SQUID}

The principle of a Smart SQUID is illustrated in Fig. 2. The voltage pulses generated by the signal and reference SQUIDs are used as the input for a digital counter which has the role of the integrator in a conventional FLL. Each relaxation oscillation of the signal SQUID or reference SQUID causes the counter output $Y$ to be increased respectively decreased 


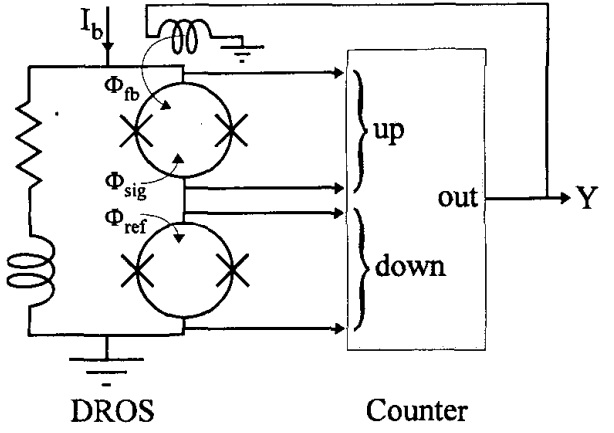

Fig. 2. Principle of a smart SQUID.

with one quantization unit $\delta Y$. The output of the counter is fed back to the signal SQUID of the DROS; the counter's quantization unit $\delta Y$ corresponds to a step $\delta \Phi_{\mathrm{fb}}$ in the feedback flux.

The system will adjust itself to the situation where both SQUIDs have the same probability to switch to the voltage state with each subsequent relaxation oscillation. If the signal flux $\Phi_{\text {sig }}$ is changed, the system is forced out of this dynamic equilibrium, and consequently only one of the SQUIDs takes part in the relaxation oscillations, which causes the counter output $Y$ to be slewed until a new dynamic equilibrium is reached. Thus, changes in the input flux $\Phi_{\text {sig }}$ result in a proportional change of the output $Y$. The Smart SQUID behaves like a digital SQUID using the relaxation oscillations as an internal clock.

If the feedback quantization step is small enough (i.e. $\delta \Phi_{\mathrm{fb}}$ « $\sqrt{ } S_{\Phi} \cdot \sqrt{ } f_{\mathrm{RO}}$, with $\sqrt{ } S_{\Phi}$ the flux noise of the bare DROS), this delta-modulated feedback system will not cause additional ("digital") noise, and the sensitivity of the system is entirely determined by the DROS.

\section{Josephson Counter}

The counter can be implemented in either semiconducting or in superconducting electronics. Since the counter should be integrated on the same chip as the DROS, it is natural to use the same (i.e. superconducting) technology for both. Instead of a complex digital counter, we chose the relatively simple "Josephson Counter" that is depicted schematically in Fig. 3.

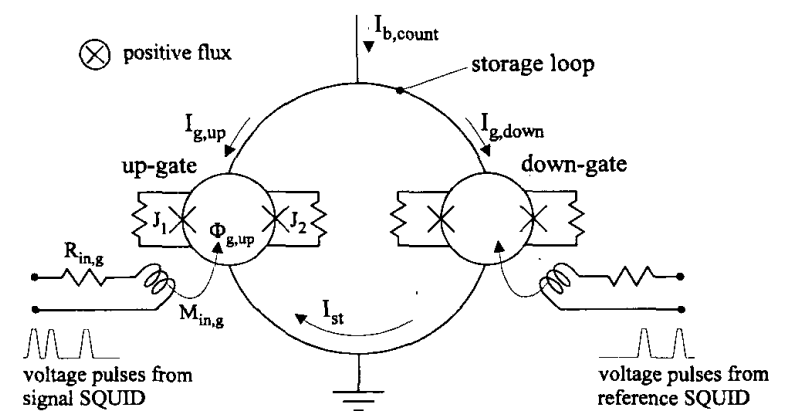

Fig. 3. Scheme of a Josephson counter.
The essence of this counter - which resembles the counters that other groups use for their digital SQUIDs - is that the voltage pulses generated by the DROS are converted to flux pulses in two write gates (up and down gate). These gates are non-hysteretic dc SQUIDs which act as "revolving doors" through which flux quanta are added or subtracted from a superconducting loop with inductance $L_{\text {count }}$.

Each added or removed flux quantum changes the circulating current in the counter, $I_{\mathrm{st}}$, by an amount of $\delta I_{\text {st }}=\Phi_{0} / L_{\text {count. }}$. The range over which $I_{\text {st }}$ can vary is determined by the operation criterion $0<I_{\mathrm{g} \text {,up }}$, $I_{g, \text { down }}<I_{\text {c,gate }}\left(\Phi=1 / 2 \Phi_{0}\right)$. Theoretically, the maximum range results at a counter bias current of $I_{\mathrm{b} \text {, count }}=I_{\mathrm{c}}\left(\Phi=1 / 2 \Phi_{0}\right)$.

By leading $I_{\text {st }}$ through the feedback coil on top of the signal SQUID of the DROS, the counter applies the feedback flux to the DROS with a quantization unit $\delta \Phi_{\mathrm{fb}}=\delta I_{\mathrm{st}} \cdot M_{\mathrm{fb}}=\Phi_{0} \cdot M_{\mathrm{fb}} / L_{\text {count }}$, where $M_{\mathrm{fb}}$ represents the mutual inductance between the signal SQUID and the feedback coil.

For the prototype Smart SQUID sensor, we used an additional non-hysteretic dc SQUID for the counter readout. Of course, this limits the versatility of the system (it still needs external FLL circuitry for the readout SQUID), but the main goal of the present experiments was to demonstrate the operation principle of a Smart SQUID.

\section{NUMERICAL SIMULATIONS OF A SMART SQUID}

Before starting the design and fabrication of the smart SQUID, the dynamic behaviour of the device was investigated by means of numerical simulations. For that purpose, the schemes of the Fig. 2 and 3 were implemented in the numerical simulation program JSIM [8].

The parameter values of the signal SQUID are: critical current of a junction $I_{0}=30 \mu \mathrm{A}$ and damping resistance $2 R_{\mathrm{d}}$ $=60 \Omega$. In order to speed up the simulations, we took a reference junction instead of a reference SQUID. The parameters of that junction are $I_{0}=45 \mu \mathrm{A}$ and $R_{\mathrm{d}}=30 \Omega$. The R-L shunt of the DROS consists of $R_{\mathrm{sh}}=1 \Omega$ and $L_{\mathrm{sh}}=10$ $\mathrm{nH}$. The time constant of the DROS, $L_{\mathrm{sh}} / R_{\mathrm{sh}}=10 \mathrm{~ns}$, implies a relaxation frequency of $f_{\mathrm{RO}} \approx 100 \mathrm{MHz}$. The values of $M_{\mathrm{in}, \mathrm{g}}=480 \mathrm{pH}$ and $R_{\mathrm{in}, \mathrm{g}}=80 \Omega$ imply an amplitude of $-2 \Phi_{0}$ for the flux pulses applied to the write gates. As $R_{\mathrm{in}, \mathrm{g}}>R_{\mathrm{d}}$, the operation of the DROS is not strongly influenced by the readout circuitry. The SQUIDs in the counter have the same junctions as those of the signal SQUID. A shunt resistance of $2 \Omega$ is used to remove the hysteresis $\left(\beta_{C} \approx 0.2\right)$. The inductance of all SQUIDs is $60 \mathrm{pH}$ and the junction capacitance is $0.5 \mathrm{pF}$. The storage loop inductance is taken as $50 \mathrm{nH}$. This small value is chosen to reduce the simulation time. The mutual feedback inductance $M_{\mathrm{fb}}$ is $480 \mathrm{pH}$.

Fig. 4 shows a numerical simulation of an operating smart SQUID. Initially, the system is in its dynamic equilibriurn, and the reference junction and the signal SQUID pulse alternately. Consequently, the current in the counter oscillates around an average value. At $t=100 \mathrm{~ns}$, a flux step of $0.2 \Phi_{0}$ is applied, disturbing the dynamic equilibrium of the system. 


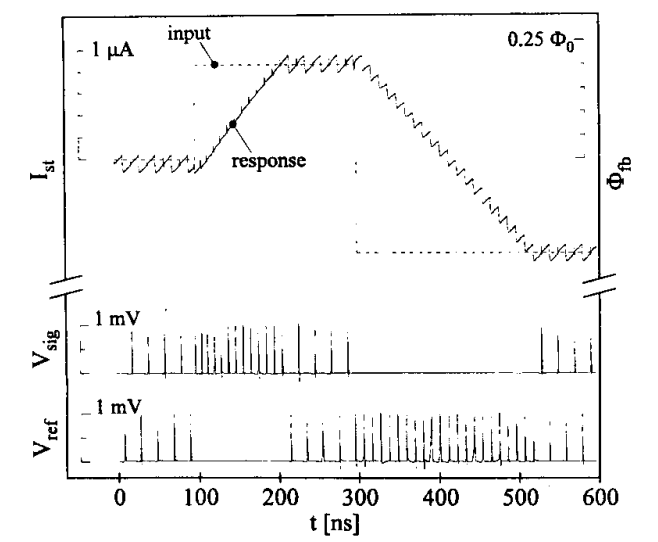

Fig. 4. Numerical simulations of the response of a smart SQUID to variations of the input flux.

As a result, only the signal SQUID produces pulses, causing the circulating current in the counter - and accordingly the feedback flux - to increase steadily until the system has established a new dynamic equilibrium. At $t=300 \mathrm{~ns}$, a flux step of $-0.4 \Phi_{0}$ is applied and now only the reference SQUID generates pulses in order to restore the dynamic equilibrium.

The simulations in Fig. 4 show that the output of the smart SQUID tracks the input flux, which indicates proper operation of the device.

\section{EXPERIMENTAL STUDY OF THE SMART SQUID}

\section{A. Chip Layout}

To investigate the smart SQUID in practice, a fully operational prototype was designed, fabricated and characterized. The design parameters were chosen on the basis of the numerical simulations discussed in the preceding section. Fig. 5 shows a micrograph of a complete integrated sensor. The devices, having a minimum feature size of $4 \mu \mathrm{m}$, were fabricated using our standard $\mathrm{Nb} / \mathrm{AlO}_{\mathrm{x}}$ thin film process. For testing purposes, also separate elements of the smart SQUID, such as the DROS, the Josephson counter or

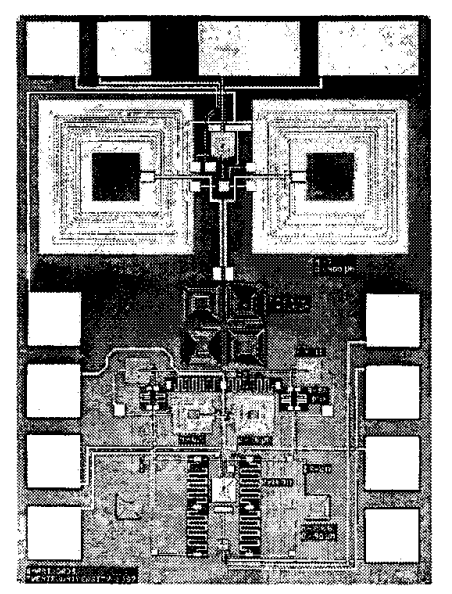

Fig. 5. Micrograph of the smart SQUID sensor. the readout SQUID, were fabricated on the same 2" wafer as the compound devices.

Both the readout SQUID and the DROS are magnetically coupled to the counter via intermediate flux transformers. Thanks to the gradiometric layout of these flux transformers, fluctuations in the bias current of the counter, $I_{\mathrm{b}, \text { count, }}$, did not induce strong flux variations in the DROS or in the readout SQUID. The effective magnetic coupling between the circulating current in the counter and the signal SQUID of the DROS was measured to be $M_{\mathrm{fb}, \text { eff }}=\partial \Phi_{\mathrm{fb}} / \partial I_{\mathrm{st}}=0.15 \mathrm{nH}$, and the coupling with the readout SQUID is $M_{\text {read,eff }}=\partial \Phi_{\text {out }} / \partial I_{\text {st }}=0.60 \mathrm{nH}$. The inductance of the storage loop of the counter was measured to be $L_{\mathrm{st}}=840 \mathrm{nH}( \pm 5 \%)$. Two resistors of $200 \Omega$ suppress the current spikes which are induced in the counter by the relaxation oscillations in the DROS. The design value of the DROS time constant, $L_{\mathrm{sh}} / R_{\mathrm{sh}}=10 \mathrm{~ns}$, implies a relaxation frequency of $f_{\mathrm{RO}} \approx 100 \mathrm{MHz}$, which operation frequency has been confirmed experimentally.

\section{B. Measurements}

Four smart DROSs have been characterized experimentally. The devices were shielded from external noise sources by means of a superconducting $\mathrm{Nb}$ shielding, and all electrical connections between the room temperature electronics and the smart DROSs were low pass filtered for RFI suppression. The readout SQUID was operated in a simple flux locked loop based on direct voltage readout without flux modulation.

In Fig. 6, the measured transfer function of one of the smart DROSs is shown for nine different values of the counter bias current $I_{\mathrm{b} \text {,count }}$. The horizontal axis shows the applied signal

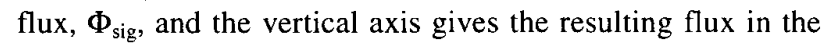
readout SQUID, $\Phi_{\text {out }}$.

The linear relation between $\Phi_{\text {sig }}$ and $\Phi_{\text {out }}$ clearly shows that the device works correctly. As expected theoretically, the linear range of the smart SQUID depends on the value of the counter bias current. At the optimum counter bias current of $I_{\mathrm{b}, \text { count }} \approx 50 \mu \mathrm{A}$, the smart DROS showed a linear response over a $\Phi_{\text {sig }}$ range of almost $3 \Phi_{0}$.

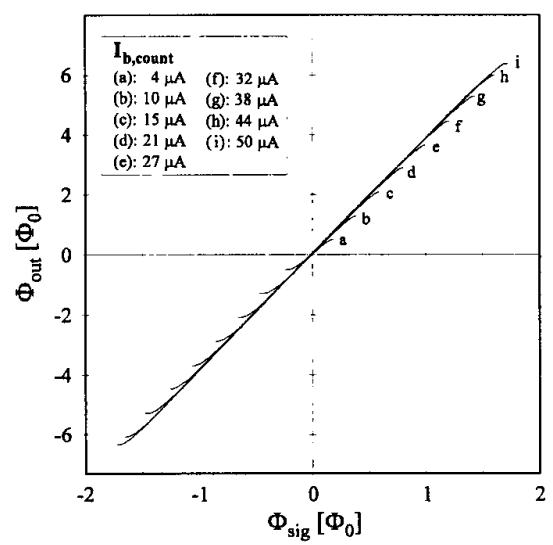

Fig. 6. Response of a smart SQUID. 


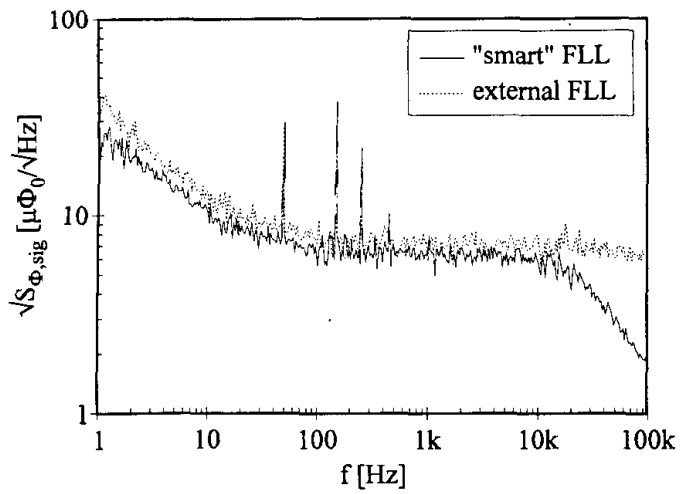

Fig. 7. Noise spectra of the smart SQUID.

The slope of the characteristics in Fig. 6 is $\partial \Phi_{\text {out }} / \partial \Phi_{\text {sig }}=3.9$. The value of $\partial \Phi_{\text {oul }} / \partial \Phi_{\text {sig, }}$, averaged over all four of the characterized devices, was $\left\langle\partial \Phi_{\mathrm{ou}} / \partial \Phi_{\mathrm{sig}}\right\rangle=3.95$, with a maximum deviation of $\pm 2 \%$, which is in good agreement with the value of $M_{\text {read,eff }} / M_{\mathrm{fb} \text {,eff }}=4.0$.

Trace (a) of Fig. 7 shows the experimental flux noise spectrum of one of the characterized devices. The white flux noise level of $\sqrt{ } S_{\Phi \text {. sig }} \approx 6.5 \mu \Phi_{0} / \sqrt{ } \mathrm{Hz}$ corresponds to an energy sensitivity of $\varepsilon \approx 2000 h$, where $h$ is the constant of Planck. Subtracting the noise contribution of the readout SQUID, the intrinsic flux noise level of the smart SQUID was deduced to be $-5 \mu \Phi_{0} / \sqrt{ } \mathrm{Hz}\left(\varepsilon_{\text {intr }} \approx 1150 h\right)$.

Trace (b) of Fig. 7 shows the flux noise of the same DROS, but now operated in a conventional (room temperature) flux locked loop. For this measurement, the DROS and the counter were disconnected by scratching the resistors $R_{\mathrm{in}, \mathrm{g}}$ mechanically. The measured white flux noise, $7 \mu \Phi_{0} / \sqrt{ } \mathrm{Hz}$, is almost identical to the overall flux noise $\left(\sim 6.5 \mu \Phi_{0} / \sqrt{ } \mathrm{Hz}\right)$, and somewhat above the intrinsic flux noise $\left(\sim 5 \mu \Phi_{0} / \sqrt{\mathrm{Hz}}\right)$ of the smart SQUID. Thus, the intrinsic flux noise of the DROS in the smart configuration is even better than in the conventional configuration. The reason for this could be that the resistors $R_{\text {in.g }}$, which are connected in parallel to the damping resistors $R_{\mathrm{d}}$ of the DROS, improve the damping of the parasitic $L_{\mathrm{sh}}-C_{\mathrm{sq}}$ resonances.

The bandwidth and the slew rate of the smart DROS prototype could not be determined, since these performance parameters were limited by the flux locked loop circuitry of the readout SQUID, having a band width of about $20 \mathrm{kHz}$.

\section{DISCUSSION AND CONCLUSIONS}

The results in Section IV show that the smart DROS works in practice as well as in theory. The energy sensitivity of the presented prototype, $\varepsilon \approx 2000 h\left(\varepsilon_{\text {intr }} \approx 1150 h\right)$, is better than that of the original Fujitsu single-chip SQUID almost by a factor of two. However, the presented prototype still needs some fine-tuning to become a real practical device.

For instance, the relaxation frequency could be increased to
$-1 \mathrm{GHz}$ in order to increase the sensitivity, the bandwidth and the slew rate. Furthermore, a new design for the storage inductor and the intermediate flux transformers can improve the dynamic range of the device considerably.

However, these modifications do not eliminate the bottleneck of the prototype, constituted by the readout SQUID. Because of this readout SQUID, the initial aim of the smart DROS, viz. operation without external flux locked loop electronics, has not been accomplished yet. At present, we are studying alternative readout schemes to get around this situation.

The smart DROS could for instance be read out in the same way as the Fujitsu single-chip SQUID by counting the output voltage pulses of the DROS with a room temperature bidirectional counter. Cryogenic pre-amplification of the voltage pulses, for instance with a high electron mobility transistor (HEMT), might be necessary in this case.

As mentioned in Section III(C), the counter of the smart DROS could also be implemented using semiconductor electronics. A smart DROS based on such a semiconducting counter would generate an output voltage which varies linearly with the input flux. As it is not straightforward to integrate the superconducting and the semiconducting elements of such a "hybrid" smart DROS on a single chip, it would consist of several discrete components, which increases the system complexity. However, the number of wires between room temperature and the SQUIDs in a multichannel system could be reduced drastically with this configuration.

Further development of the smart DROS, following the recommendations above, offers the perspective of a very sensitive, fast and versatile SQUID sensor.

\section{REFERENCES}

[1]N. Fujimaki, H. Tamura, T. Imamura, and S. Hasuo, "A single-chip SQUID magnetometer", IEEE Trans. Electron Devices 35, pp. 2412-2418, 1988.

[2] M. Radparvar, "A wide dynamic range single-chip SgUID magnetometer", IEEE Trans. Appl. Supercond. 4, pp. 87-91, 1994.

[3] U. Fath, R. Hundhausen, T. Fregin, P. Gerigk and W. Eschner, "Experimental digital SQUID with integrated feedback circuit", IEEE Trans. Appl. Supercond. 7, pp. 2747-2751, 1997.

[4] S.V. Rylov, "Analysis of high-performance counter-type A/D converters using RSFQ logic/memory elements", IEEE Trans. Magn. 27, pp. 2431-2434, 1991.

[5] Fujitsu Laboratories Ltd., 10-1 Morinosato-Wakamiya, Atsugi 243-01, Japan.

[6] D.J. Adelerhof, M.J. van Duuren, J. Flokstra and H. Rogalla, "High sensitivity magnetic flux sensors with direct voltage readout: Double relaxation oscillation SQUIDs", IEEE Trans. Appl. Supercond. 5, pp. 2160-2163, 1995

[7] M.J. van Duuren, D.J. Adelerhof, G.C.S. Brons, J. Kawai, G. Uehara, H. Kado, J. Flokstra and H. Rogalla, "Frequency readout of relaxation oscillation superconducting quantum interference devices in the $\mathrm{GHz}$ regime", J. Appl. Phys. 80 , pp. 4164-4173, 1996.

[8] Simulation software: JSIM 2.1, developed by E.S. Fang, University of California, Berkeley, CA 94720 , U.S.A. 